\title{
L'accréditation: renouvellement du mandat de formation postgraduée
}

\section{Werner Bauer}

Dr med., président de I'Institut suisse pour la formation médicale postgraduée et continue ISFM

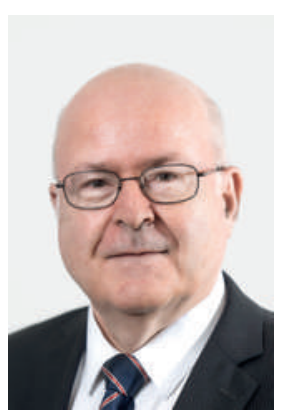

Avec l'introduction de la reconnaissance réciproque des diplômes en Europe en 2002, les titres de spécialiste de droit privé sont devenus des titres fédéraux. Pour la FMH et les sociétés de discipline médicale, il était essentiel que la mise en application des dispositions légales (loi sur les professions médicales) soit confiée à une organisation médicale et que les médecins restent responsables de l'organisation de leur formation postgraduée. Ce fut le cas avec la création de l'Institut suisse pour la formation médicale postgraduée et continue (ISFM), nouvel organe de la FMH à qui cette mission a été confiée. Pour cela, l'ISFM édicte la Réglementation pour la formation postgraduée et gère les différents programmes de formation aux côtés des sociétés de discipline. Ces programmes servent ensuite de base à la reconnaissance des établissements de formation, aux visites d'établissements et à l'octroi des titres de spécialiste. Mais la Confédération ne délègue pas ces compétences uniquement sur une base de confiance ni pour une durée illimitée. Les organisations comme l'ISFM et les sociétés de discipline qui assument une tâche de l'Etat sont régulièrement évaluées par le Département fédéral de l'intérieur (DFI) à l'occasion d'une procédure d'accréditation.

L'ISFM, de même que toutes les sociétés de discipline en charge d'un titre de spécialiste, sont soumis à cette évaluation tous les sept ans: une procédure de longue haleine particulièrement coûteuse, fondée sur un rapport d'auto-évaluation qui contient toute une série de questions sur différents "standards de qualité».

Il s'agit également de répondre aux exigences suivantes: Action autonome dans les situations d'urgence. Prise de responsabilités dans le domaine de la santé. L'évaluation se fonde sur des méthodes formatives et sommatives, auxquelles vient s'ajouter un feedback régulier. La formation postgrade est régulièrement soumise à des évaluations par les formateurs, ainsi que par les personnes en formation. Un plan de mesures est esquissé pour l'avenir (développement de la qualité de la filière de formation postgrade).

Les rapports sont soumis à une équipe d'experts composée de membres de chaque spécialité médicale qui étudient les dossiers de leur discipline respective et tentent de clarifier les questions en suspens lors d'une table ronde. Les experts rédigent ensuite leurs rapports sur la base du rapport d'auto-évaluation et de leur discussion, rapports qui peuvent ensuite être commentés par la Commission des professions médicales (MEBEKO). En cas de nécessité avérée, l'accréditation peut être liée à des recommandations ou conditions particulières. La procédure est organisée par l'Agence suisse d'accréditation et d'assurance de la qualité (AAQ) et chapeautée par l'Office fédéral de la santé publique (OFSP) et par le DFI qui met ensuite en vigueur ces dispositions.

Pourquoi un éditorial sur l'accréditation? Trois raisons à cela:

- Le modèle suisse de réglementation et de réalisation de la formation postgraduée est remarquablement

\section{L'accréditation est l'occasion de mener une} réflexion approfondie sur l'avenir de la formation médicale.

simple et efficace, et illustre parfaitement la bonne collaboration entre une organisation professionnelle privée (ISFM) et une institution étatique (OFSP).

- L'accréditation représente une grande charge de travail pour les sociétés de discipline, qui travaillent selon un système de milice. Elle n'est donc pas toujours bien acceptée et suscite parfois incompréhension et irritation. A ce propos, il est important de rappeler que la liberté avec laquelle les médecins peuvent organiser la formation postgraduée va de pair avec des obligations et des devoirs auxquels nous ne pouvons pas nous soustraire. Par ailleurs, l'accréditation prend encore plus de sens si nous en profitons pour mener une réflexion approfondie sur l'avenir de la formation médicale.

- Enfin, nous souhaitons inviter l'AAQ et l'OFSP à mener la procédure d'accréditation de la manière la plus simple et efficace possible, et à tout mettre en œuvre pour nommer des experts compétents. En effet, de ces derniers dépend l'issue de la procédure, à savoir si l'accréditation a réellement porté ses fruits ou si elle n'a été réduite qu'à une simple procédure administrative; ce dont personne ne voudrait. 